\title{
Combined Heat and Power Dynamic Economic Dispatch incorporating pumped hydro energy storage considering uncertainty and outage of renewable energy sources
}

\author{
Chitralekha Jena ${ }^{1}$ \\ ${ }^{1}$ Kalinga Institute of Industrial Technology
}

January 14,2022

\begin{abstract}
Owing to increasing penetration of renewable energy sources, it is mandatory to investigate it's effect on the combined heat and power dynamic economic dispatch. At the same time, adverse effect is there due to highly intermittent nature and higher rate of outages of these sources. This piece of work proposes squirrel search algorithm (SSA) for solving combined heat and power dynamic economic dispatch (CHPDED) incorporating pumped-storage-hydraulic unit captivating uncertainty and outage of renewable energy sources. A lately developed swarm intelligence algorithm SSA, emulates from the dynamic scavenging behavior of squirrel. The competence of the recommended technique is examined on a test system. Simulation outcomes of the proposed technique is harmonized with those acquired by particle swarm optimization (PSO) and grey wolf optimization (GWO). After comparison, a conclusion was made presenting SSA technique conferring with good-quality solution than other techniques.
\end{abstract}

